\section{P382 FACTORS AFFECTING HEPATITIS C CARE IN PRISONS IN ENGLAND: A QUALITATIVE ANALYSIS OF STAKEHOLDERS IN LONDON AND ENGLAND}

Sophie Candfield*, Emma Plugge, Maciej Czachorowski, Eamonn O’Moore. National Health and Justice Team, Premier House, Reading, UK

\subsection{6/sextrans-2019-sti.479}

Background NHS England is committed to eliminating Hepatitis $\mathrm{C}(\mathrm{HCV})$ virus infection as a public health problem by 2025. Prisons are a key setting for any elimination strategy due to the high prevalence of infection among prisoners, often due to injecting drug use (IDU). During 2018 we sought to understand barriers and enablers of acceptable HCV care to incarcerated people.

Methods We used a purposive sampling strategy to identify and recruit key stakeholders along the HCV care cascade in English prisons. We interviewed key stakeholders including commissioners, healthcare providers and patient advocates. The semi-structured interviews were recorded, transcribed, and analysed thematically. PHE Research Ethics Group approved the project.

Results Sixteen individuals were interviewed including nurses/ doctors (primary and secondary care), commissioners in prisons and community settings, third sector organisations, an exincarcerated person, and laboratory personnel. Nine were based in London. Participants identified a number of important barriers. These included: Resource challenges (staffing levels, completing demands), care pathway issues (eg. implementation of testing, high patient turnover, continuity across prisons and to the community, patient nonattendance at clinic, overdependence on a single individual to ensure that the pathway functioned, and lack of reflex HCV RNA testing in laboratories), and patient and staff perceptions (eg. stigma, misunderstanding DAA side effects, cultural issues towards health and HCV). They also identified enablers that included: Providing resources (presence of a designated/paid champion), Senior support including from Governors (allowing stigma, patient attendance and pathway issues to be addressed), Collaboration (between prisons, personnel in prison, and community organisations), Peer to peer education and support, and a Focus on fail-safes in the pathway including effective planning for release or transfer.

Conclusion Prisons could be an important setting for HCV elimination strategies but multiple barriers exist to achieving model care pathways for $\mathrm{HCV}$ in prison. This research has identified areas of good practice and important areas for improvement.

Disclosure No significant relationships.

\section{P384 EXTENSION FOR COMMUNITY HEALTHCARE OUTCOMES (ECHO) IMPLEMENTED FOR HEPATITIS C (HCV)/HIV CO-INFECTED PATIENTS IN TEXAS, USA}

\footnotetext{
${ }^{1}$ Waridibo Allison*, ${ }^{2}$ Julie Parish Johnson, ${ }^{2}$ Andrea Rochat, ${ }^{1}$ Keito Kawasaki, ${ }^{2}$ Raudel Bobadilla, ' ${ }^{1}$ James Bridges, ${ }^{1}$ Trisha Melhado. ${ }^{1}$ UT Health San Antonio, Department of Medicine - Division of Infectious Diseases, San Antonio, USA; ${ }^{2}$ UT Health San Antonio, Research to Advance Community Health Center, San Antonio, USA
}

10.1136/sextrans-2019-sti.480

Background HCV prevalence in people living with HIV (PLWHIV) is approximately $25 \%$ and progression of liver disease is accelerated in PLWHIV. ECHO is a telementoring "hub and spoke" model based on knowledge-sharing networks led by expert teams using videoconferencing for sessions with community healthcare providers. We describe development and implementation of an HIV/HCV ECHO to educate/support primary care and infectious disease (ID) providers to treat HCV in PLWHIV.

Methods Development began in October 2017. The expert panel assembled consisted of: ID specialist, hepatologist, psychiatrist, addiction medicine specialist, pharmacist, community health worker. An HIV/HCV didactic curriculum was developed. Initial spoke sites were providers at five HIV clinics. Equipment set up was ensured at the hub site. Minimal hardware requirement for spoke participation was a smart phone. A project website was developed. Innovations in use of REDCap, a cloud database platform, were developed. UT Health San Antonio HIV/HCV ECHO launched 1st October 2018.

Results One-hour ECHO sessions are held twice a month. The HIV/HCV curriculum covers 11 topics. From 1st October 2018 - 22nd January 2019, 158 individuals participated in sessions including clinicians responsible for 1,155 PLWHIV, 42 co-infected with HCV. REDCap innovations included direct entry of de-identified clinical information for sessions into REDCap with export into a PDF format, automatic assignment of ECHO identification numbers for initial and follow up case presentations, direct entry of information required for Continuing Medical Education credit into REDCap. Website views were 1887 with 253 individuals using the site.

Conclusion ECHO has been an effective foundation for a growing Community of Practice and Learning for $\mathrm{HIV} / \mathrm{HCV}$ management. REDCap innovations facilitate ECHO administration. Reach to HIV/HCV co-infected patients has been demonstrated. The ECHO model should be considered to improve education about HCV management among HIV providers and to improve access to HCV treatment and cure for PLWHIV in their medical home.

Disclosure No significant relationships.

\section{P386 CANCER RISK AMONG PEOPLE WITH HIV, HBV AND/OR HCV INFECTIONS}

${ }^{1}$ Maryam Darvishian*, ${ }^{2}$ Carmine Rossi, ${ }^{3}$ Stanley Wong, ${ }^{3}$ Amanda $\mathrm{Yu},{ }^{3}$ Jason Wong, ${ }^{1} J a n e$ Buxton, ${ }^{3}$ Mark Gilbert, ${ }^{3}$ Mawuena Binka, 'Zahid Butt, ${ }^{1}$ Sofia Bartlett, 'Margo Pearce, ${ }^{3}$ Maria Alvarez, ${ }^{3}$ Darrel Cook, ${ }^{3}$ Troy Grennan, ${ }^{4}$ Ryan Woods, ${ }^{4}$ John Spinelli, ${ }^{1}$ Mark Tyndall, ${ }^{5}$ Mel Krajden, ${ }^{3}$ Naveed Janjua. 'University of British Columbia, Vancouver, Canada; ${ }^{2} B C$ Centre for Disease Control, Clinical Prevention Services, Vancouver, Canada; ${ }^{3}$ British Columbia Centre for Disease Control, Vancouver, Canada; ${ }^{4} B C$ Cancer, Vancouver, Canada; ${ }^{5} B C$ Centre for Disease Control, Vancouver, Canada

10.1136/sextrans-2019-sti.481

Background HIV, hepatitis B virus (HBV) and hepatitis C virus (HCV) infections each are associated with increased cancer risk. In this study, we assessed the effect of co-occurrence of HIV, HBV and HCV on all cancers, anal cancer, non-Hodgkin Lymphoma (NHL) and liver cancer.

Methods We used the British Columbia Hepatitis Testers Cohort (BC-HTC) which includes all individuals $(\sim 1.7$ million) tested for HCV or HIV, or diagnosed with HCV, HIV, or HBV linked with data on cancers. We included individuals tested for all three infections since 1990 and followed them 